\title{
Guest editorial special issue on quality of experience for multimedia applications
}

\author{
Mikołaj I. Leszczuk • Eduardo Cerqueira • \\ Marília Curado • Andreas Mauthe • Sherali Zeadally
}

Published online: 18 July 2012

C The Author(s) 2012. This article is published with open access at Springerlink.com

In recent years, the ubiquity of multimedia services along with the proliferation of mobile devices and the demand for new audio and video applications are changing the lifestyle of users. The multimedia era is allowing users to create, distribute, and access content in ubiquitous way and cost-effectively, while providers explore new ways to increase their revenues. The efficient delivery of real-time multimedia services over emerging diverse and heterogeneous systems is a challenging research goal. The interoperability of applications, transport and network protocols as well as the demand for improved Quality of Experience (QoE) create new challenges and opportunities for further research on novel communication protocols, architectures, and methods to efficiently support current and future multimedia networking systems.

In 2010, the Third International Future Multimedia Networking (FMN 2010) workshop was organized in Kraków, Poland and achieved a success attracting numerous submissions from various countries. FMN 2010 produced a high quality peer reviewed technical programme with an acceptance rate at around $24 \%$ and addressed important aspects of future multimedia systems with a focus on QoE.

Authors of selected papers from FMN 2010 were invited to submit extended versions of their papers in order to be considered for inclusion in this Special issue on Future Multimedia.

M. I. Leszczuk $(\bowtie)$

AGH University of Science and Technology, Krakow, Poland

e-mail: leszczuk@agh.edu.pl

E. Cerqueira

Federal University of Para, Para, Brazil

M. Curado

University of Coimbra, Coimbra, Portugal

A. Mauthe

Lancaster University, Lancaster, UK

S. Zeadally

University of the District of Columbia, Washington, DC, USA 
These papers were peer-reviewed again and then further revised by the authors.

The paper 'Standard Multimedia Conferencing in the wild: the Meetecho Architecture' presents a conferencing architecture called Meetecho. The paper embraces an engineering approach, by describing an actual implementation of an open source centralized videoconferencing system capable of offering an advanced communication experience to endusers through the effective exploitation of mechanisms such as session management and floor control. Meetecho has been designed to be fully compliant with the latest standard proposals coming from both the IETF and the $3 \mathrm{GPP}$ and can be considered as an outstanding example of a real-time application built around the SIP protocol. The paper discusses both the design of the overall conferencing framework and the most important issues faced during the implementation phase.

The paper 'QoS-RRC: An Overprovisioning-centric and Load Balance-aided Solution for Future Internet QoS-oriented Routing' introduces the Quality of Service (QoS)-Routing and Resource Control (QoS-RRC), a set of Generic Path (GP)-compliant facilities. QoS-RRC complements GP architecture with QoS-oriented routing, with the aid of load balancing to select paths that comply with session-demands while keeping residual bandwidth to increase user experience. To address scalability issues, QoS-RRC operates on the basis of an overprovisioning-centric approach to achieve cost-effectiveness in terms of state storage, signaling load and network operations. An initial QoS-RRC performance evaluation was carried out in Network Simulator v.2 (NS-2), which showed that there had been drastic improvements in the flow delay experience and bandwidth use over a range of relevant stateof-the-art solutions. Moreover, the impact of QoS-RRC on the user experience (compared to current IP QoS and routing standards) has been evaluated, by analyzing the main objective and subjective QoE metrics, namely Peak Signal to Noise Ratio (PSNR), The Structural Similarity Index (SSIM), Video Quality Metric (VQM) and Mean Opinion Score (MOS).

The paper 'A Fuzzy Queue-aware Routing Approach for Wireless Mesh Networks' presents a variation of the Wireless Mesh Network (WMN) routing protocol Optimized Link State Routing (OLSR), to achieve QoE requirements for multimedia applications. It is based on the dynamic choice of metrics and in a Fuzzy Link Cost (FLC) to determine the best routes for multimedia packets. Simulations were carried out to show the benefits of the proposed metric regarding user experience compared to existing versions of OLSR.

The paper 'Content Driven QoE Assessment for Video Frame Rate and Frame Resolution Reduction' presents two No-Reference (NR) metrics mapping frame rate or resolution into MOS. Both models use simple to calculate parameters expressed by sequence spatial and temporal information. The models were estimated and verified upon distinctive video sequence sets. The considered frame rate change varies from 5 to 30 frames per second. The considered resolutions changes from Sub Quarter Common Intermediate Format (SQCIF) to Standard Definition (SD).

The paper 'Framework for the Integrated Video Quality Assessment' addresses the design challenges of an integrated framework using a number of comprehensive functional modules. The framework integrates objective quality assessment models of Artifacts Measurement (AM) and Quality of Delivery (QoD) approaches. Only the best fit models are activated by the framework considering requirements of individual evaluation tasks. The paper also introduces the recent work of realizing key functional modules of the framework. Joint subjective experiments between two institutes have also been carried out to evaluate the implementation. Experimental results demonstrate the concept of an integrated framework and show the effectiveness of its key modules in estimating the quality level of video services. 
In the paper 'An Adaptive Motion-Compensated Approach for Video De-Interlacing', an adaptive, edge-preserving motion-compensated approach for video de-interlacing is proposed. The algorithm preserves strong edges and interpolates the missing pixels along the contours depending on the motion-degree of the region to which they belong. The proposal is optimized to reduce heavy computation, which is the main drawback of motion-compensated deinterlacing algorithms. Therefore it provides complexity scalability as a trade-off tool between performance and computation time. Experiments demonstrate a significant gain in reconstruction quality as compared to other de-interlacing implementations.

We wish to thank all the authors for considering this special issue of Multimedia Tools and Applications Journal as a venue for submitting their papers. We express our gratitude to all reviewers who devoted their precious time in providing valuable feedback on all the papers considered for this special issue.

Finally, we thank the Editor-in-Chief, Professor Borko Furht, for his great support and encouragements. We would also like to take this opportunity to thank Springer staff for their unconditional support and time throughout the preparation of this issue.

We hope that this special issue will provide a valuable source of reference for researchers, designers, engineers, and developers working in the area of multimedia networking.

\author{
Mikołaj I. Leszczuk \\ Eduardo Cerqueira \\ Marília Curado \\ Andreas Mauthe \\ Sherali Zeadally
}

Open Access This article is distributed under the terms of the Creative Commons Attribution License which permits any use, distribution, and reproduction in any medium, provided the original author(s) and the source are credited.

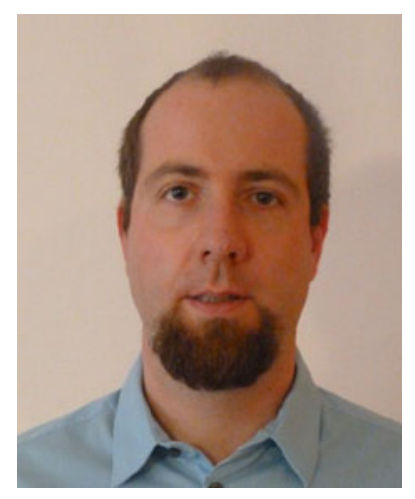

Mikolaj Leszczuk received the M.Sc. degree in electrical engineering and the Ph.D. degree in telecommunications from the AGH University of Science and Technology, Krakow, Poland, in 2000 and 2006, respectively. In 2000, he joined the Department of Telecommunications at the AGH University of Science and Technology, where he is currently an Assistant Professor. He spent his scholarship leaves at Charles III University of Madrid, Spain. He is the member of the IEEE. He has been a consultant to governmental institutions and telecom operators in modern telecommunication networks. His research interests include multimedia, QoE (Quality of Experience) and security. He has/had actively participated in eContentPlus, 
CULTURE 2000, Eureka - Celtic Plus, and 4th, 5th, 6th, 7th FP European programs (ACTS, IST, ICT, and SECURITY). He co-authored more than 100 publications including 3 books. He served as a guest editor to Springer Multimedia Tools and Applications. He has also been appointed as an expert in Information and Communications Technologies and Security by the European Commission.

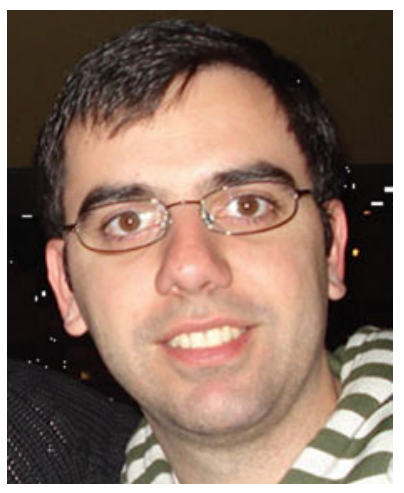

Eduardo Cerqueira received His $\mathrm{PhD}$ from University of Coimbra, Portugal, in 2005 and now is an Associated Professor at the Department of Computer Engineering of the Federal University of Para, Brazil. His research interests are Quality of Service, Quality of Experience, Multimedia, Mobility, Routing, and Resilience. He has participated in several EU and Brazilian projects, such as Q3M, SAPRA, CONTENT and REDE TIC.

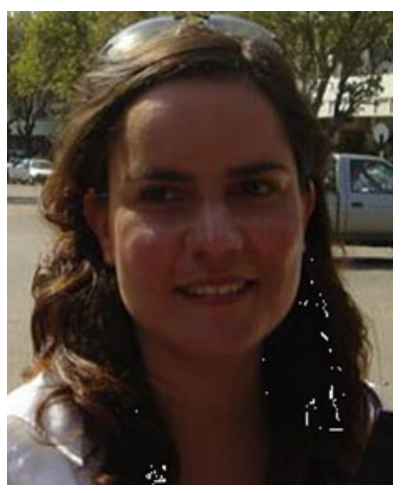

Marilia Curado is an Assistant Professor at the Department of Informatics Engineering of the University of Coimbra, Portugal, from where she got a PhD in Informatics Engineering on the subject of Quality of Service Routing, in 2005. Her research interests are Quality of Service, Mobility, Routing, and Resilience. She has participated in several projects, in Networks of Excellence from IST FP5 and FP6, in the IST FP6 Integrated Projects, EuQoS and WEIRD, and on ICT FP7 STREPs MICIE and GINSENG. 


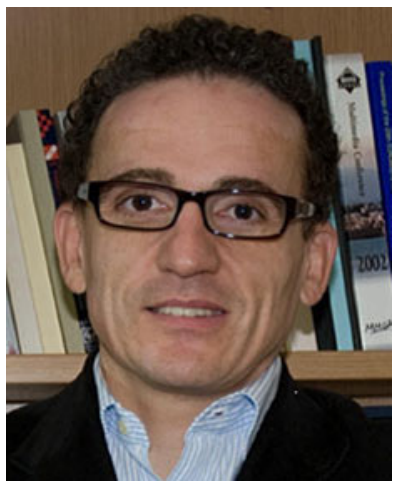

Andreas Mauthe is Senior Lecturer at the Computing Department, Lancaster University. He has been working in the area of distributed and multimedia systems for more than 15 years. His particularly interests are in the area of content management systems and content networks, large scale distributed systems, peer-topeer systems, and self-organisation aspects. Prior to joining Lancaster University, Andreas headed a research group at the Multimedia Communications Lab (KOM), at the Technical University of Darmstadt. After completing his $\mathrm{PhD}$ in Lancaster in 1997, Andreas worked for more than 4 years in different positions in industry in the area of content management in content production and media archives.

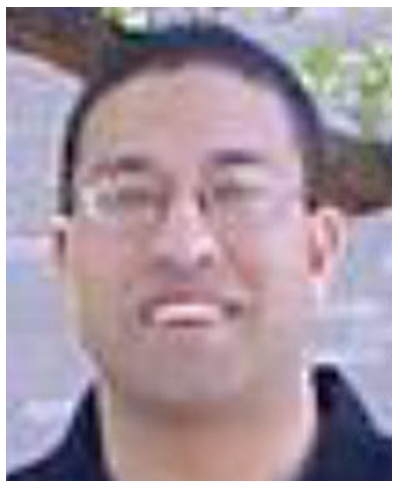

Sherali Zeadally received his Bachelor degree in Computer Science from University of Cambridge, England, and the Doctoral degree in Computer Science from University of Buckingham, England, in 1996. He is an Associate Professor at the University of the District of Columbia. He currently serves on the Editorial Boards of 15 peer-reviewed international journals. He has been serving as a Guest Editor for over a dozen special issues of various peer-reviewed scholarly journals. He is a Fellow of the British Computer Society and a Fellow of the Institution of Engineering Technology, UK. His research interests include computer networks including wired and wireless networks, network and system security, mobile computing, ubiquitous computing, RFID, multimedia, performance evaluation of systems and networks. 